\title{
The Drive Design of the STM32-based Brushless DC Motor
}

\author{
Song Wang, a , Wang Guo ${ }^{2}$, Wenqiang Dun ${ }^{2}$ \\ ${ }^{1}$ UAV Research Institute, Beihang University, Beijing, 100191, China \\ ${ }^{2}$ Robotics Institute, Beihang University, Beijing, 100191, China \\ awsongking@126.com
}

\begin{abstract}
.
This paper proposes a high-performance STM32 microprocessor issued by the STMicroelectronics for the design plan of the brushless DC motor. The special power-driven chip IR2136 is the core of the drive part. The design of the system hardware, master software, and PC software are completed. The position control adopts the PD controller based on the angular position information fusion technology. The working conditions, such as the over-voltage, over-current, overheating, and crashes, are effectively protected. Prototype tests show that the system has the desired location and follows the response effect. The system also shows high reliability.
\end{abstract}

Keywords: Brushless Motor; STM32; IR2136; Position Control; Information Fusion

\section{Introduction}

The brushless motor has been developed rapidly with the increasing growth of technology. The brushless motor is widely used in the fields of the aerospace, medical equipment, home appliances, and electrical cars because of its brushless, low interference, low noise, smooth operation, long life, low maintenance cost, and other advantages. The brushless DC motor control has evolved from using an analog control circuit to using a microprocessor as the core of a digital control circuit. Producing a high-performing microprocessor and high operation speed is the goal for designing a controller that meets the requirements of motor real-time control for application in modern industries[1].

The STM32 series issued by STMicroelectronics is based on the specially designed ARM Cortex-M3 core for high-performance, low-cost, and low-power embedded applications. With its interrupt handling fully based on the hardware, the interrupt speed is faster. The STM32 series has enhanced clock frequency up to $72 \mathrm{MHz}$. Thus, this microprocessor not only has the architecture necessary for high-speed signal processing and digital control functions, but also the peripheral equipment required to achieve a brushless motor control. It is characterized by its powerful digital processor [2], as follows: 
- $\quad$ 1.25DMips/MHz (i.e., the speed of $\mathrm{MHz}$ per second allow the performance of Dhrystone instructions, 1.25M). This characteristicenables the STM32 controller to provide a better performance than the traditional 16-bit microcontroller and microprocessor.

- With first-class peripherals, as follows: $1 \mu \mathrm{s}$ dual 12 ADC, 4 Mbit/s UART, $18 \mathrm{Mbit} / \mathrm{s} \mathrm{SPI}$, and $18 \mathrm{MHz} \mathrm{I} / \mathrm{O}$ flip speed.

- Low power consumption: the consumption is $36 \mathrm{~mA}$ at $72 \mathrm{MHz}$ (all peripherals active)and $2 \mu \mathrm{A}$ during standby.

In addition, it also has peripheral resources for motor control:

- An advanced control timer producing a complementary PWM waveform embedded into the dead-time (TIM1 and TIM8) is included.

- $\quad$ The general-purpose timer TIM2 can be used as "Interface Timer" to connect the Hall sensor.

These outstanding features of the STM32 series allow the development and design of a high-performance STM32-based brushless DC motor drive system.

\section{Hardware Design}

The system hardware is designed in a two-layer structure, which is divided into two main modules. The lower layer is the power module, and the upper layer is the control module. Each module circuit design is on separate PCBs, and the upper and lower layers can be mutually plugged. This modular design approach makes system replacement easy and fast, and the power module can also be designed in various types to drive the brush motors, brushless motors, and other motors compatible with the control module. By replacing the switch $5 \mathrm{~V}$ power supply, the support for external DC power supply at a wide voltage range(9V to $36 \mathrm{~V})$ can be implemented. Its functional block diagrams are shown in Figs.1 and 2.

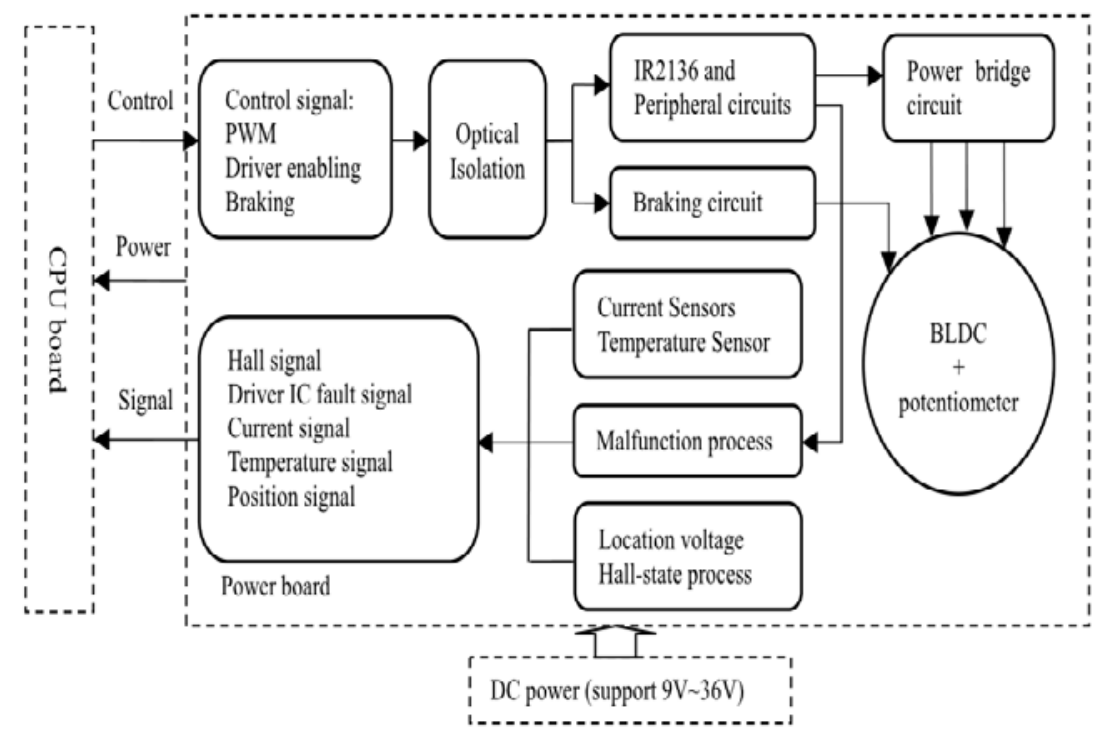


Fig.1. Structure diagram of the power module

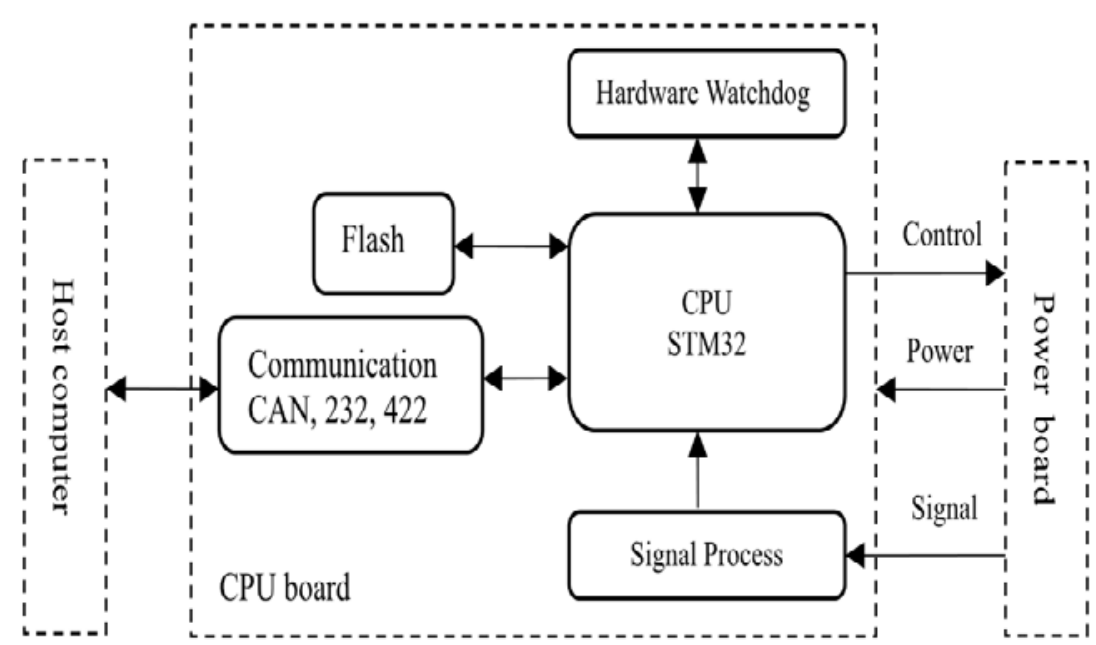

Fig.2. Structure diagram of the control module

Power Supply Design. The normal and reliable work of electrical systems depends on a stable power supply system. For the drive control of the motor, the two parts are separated completely in an isolated manner because the driving portion often interferes with the control section. Therefore, different power supplies are needed. An adjustable output voltage LDO is usedtosupply12V to the power driver chip.The independent switching power supply converts the external power supply into a 5V DC one, whereas the fixed output LDO power supply convertsthe5V voltage to $3.3 \mathrm{~V}$ to supply the CPU, external FLASH, and hardware watchdog. In addition, the high-precision analog power supply provides the voltage for location acquisition. The input and output terminals of each power module are installed along with filter capacitors.

Motor Control Implementation.STM32 series of advanced timer generates three pairs of dead-time embedded complementary PWM signals to drive the three-phase brushless DC motor. The PWM duty cycle and output polarity can be freely changed according to need. The speed of the brushless DC servo motor is mainly achieved by adjusting the duty cycle of the PWM. The selection of the PWM carrier frequency directly affects the performance of the entire control system. If the frequency is too high, the anti-interference ability is strong. Moreover, the noise of the motor can be reduced, but the power switch consumption is large. If the frequency is too low, the consumption of power tube scan be reduced, but the performance of the motor operation is low and it emits a relatively loud noise. Thus, the PWM carrier frequency range is generally in the $15 \mathrm{KHz}$ to $30 \mathrm{KHz}$ range. This system uses $18 \mathrm{KHz}$, and a PWM signal is embedded in the1.38us dead-time; these measures prevent the upper and lower bridge arms from conducting simultaneously because of interference to consume the switch tubes [3].

The drive circuit in this design has the special driver power chip IR2136 as 
the center, thereby converting the PWM signal of the control module into a signal driving the 6 MOSFET devices in the main circuit of the three phase inverter bridge;controllingthe three-phase inverter bridge to convert the DC power supply into three-phase DC voltage $\mathrm{U}, \mathrm{V}$ and $\mathrm{W}$; and driving the operation of the brushless motor to achieve the drive purposes of the drive circuit simply by entering a DC power supply.

IR2136 is a special three-phase bridge driver withthe following: three pairs of independent high side and low side output channels;outputting 6-channel output drive pulseswith operating frequency nearly reaching hundreds of thousands $\mathrm{Hz}$; a dead-time;and the typical value of 0.29us. This driver functions inunder-voltage and over-current protection and indicatesthe under-voltage and over-current fault conditions. Its input end is compatible with LSTTL and CMOS logic with a noise filter [4].

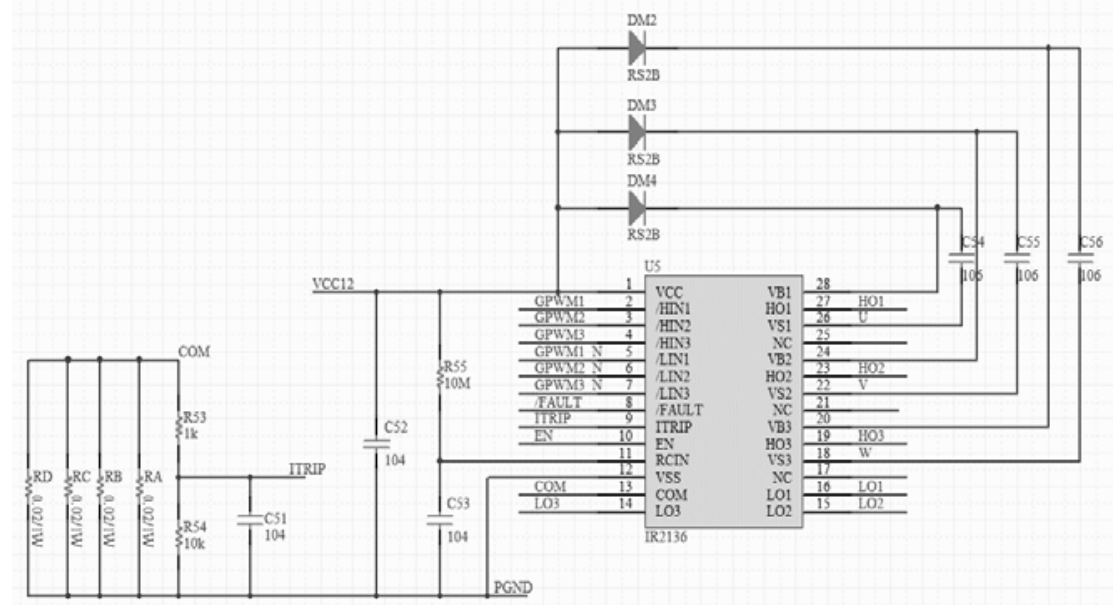

Fig.3. IR2136 and its peripheral circuit

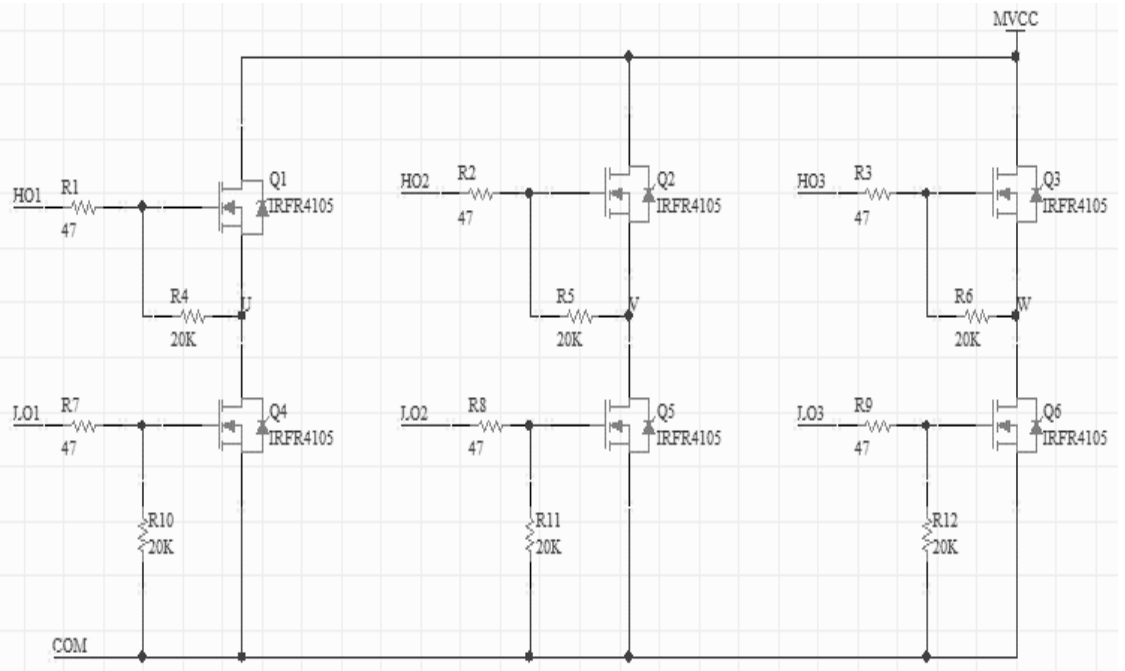


Fig.4. Three phase inverter bridge circuit

Signal Isolation and Information Collection. The digital circuit part requires relatively low operating voltage and current, whereasthe power part requires higher voltage and current. If the high voltage and largecurrent of the power drive part enterthe digital circuit, it will cause interference inthe digital circuit, thereby causing abnormal function. To ensure normal work, thedigital circuits and power-drivemust be isolated and matched. This system mainly uses optical isolation TLP281 to achieve isolation. It transmits electrical signals with light as a medium, thereby creatinggood isolation for the input and output signals Strong anti-interference ability and fast response are observed, thereby ensuring the reliability of the entiresystem. In addition, the output voltage can be adjusted through optical isolation to meet the needs of the back-end of the circuit.

The inside of the brushless DC motor for debugging in this system is embedded with three Hall position sensors, which differed by $120^{\circ}$ in space. The motor rotor is a permanent magnet when it rotates,and the magnetic field it generatedwill also rotate. Each Hall sensor will produce a $180^{\circ}$ output pulse signal. The signal outputs from the three Hall sensors mutually differ by $120^{\circ}$, thereby generating six signals that can be collected in the Hall state, as follows: 0x05,0x04,0x06,0x02,0x03, and 0x01.Each mechanical rotation has six states, and the sequence of such states can be changed by adjusting the order of the three Hall signal lines of $\mathrm{H}_{\mathrm{u}}, \mathrm{H}_{\mathrm{v}}$, and $\mathrm{H}_{\mathrm{w}}$. Thus, the advanced timer changes the switch control signal in turnto achieve the continuous rotation of the motor through the phase sequence provided by the Hall sensor.

The output signal of the Hall sensor is not stable and frequent and exhibitsinterfering signals. Thus,it is necessary to filter it before it enters the captured unit of the CPU.Afilter capacitorand six inverters 74HC04 areused. The output signal of the sensor is sent to the CPU's capture unit after two reversals, thereby improvingthe anti-jamming capability and achieving a good filtering effect.

The TIM2 of the STM32 timer can be used as "interface timer" to connect the Hall sensor. This timer hasthree timer input pins (CC1, CC2, and CC3) connected to the TI1 input channels via an XOR gate. An"interface timer" captures the signal. Wheneverone of the three inputschanges, the new counter starts counting from 0 . This produces a time reference that is triggered by any change in the Hall input, where the motor rotor position and speed information are obtained.

The output shaft of the motor is connected tothe rotary potentiometer through shaft coupling. Thepotentiometer is powered byan independent high-precision analog power supply. The wiper of thebuilt-in ADC acquisition potentiometer of the STM32 is used to output the voltage. Therefore, the absolute position of the motoris obtained.

Other Parts. This system is equipped with a 232 conversion module, 422 conversion module, and CAN bus transceiver module for a variety of communication modes. TheCAN bus has long transmission distance, strong anti-electromagnetic interference capability, and strong error detection capabilitywith several advantages, such as priority and arbitration functions. 
Thus, the system is able to communicate with the main systems only via the CAN bus after the system solidified, packed, andsecured the package to achieve the motor position control.

To monitor the built-in under-voltage over-current fault signals of the power driver chip IR2136, this system also has the following protective implements:

(a) A current sensor is added in the DC power input to monitor the total system current;

(b) A reasonable range is achieved by an external DC power supply voltage op amp treated for monitoring the external voltage of the system;

(c) due to excessive current and high voltage of the MOS tube, a temperature sensor is added into the system to regulate the MOS tube temperature to the temperature sensor through the top-dressed copper for monitoring the maximum temperature of the system;

(d) A hardware watchdog is added and is fed by the CPU in a timely manner. When some procedural errors and potential adverse environmental interferences and other factors lead to a system crash, the self-healing of the system is achieved by inputting a reset signal into the CPU in the absence of human intervention.

Aflash chip AT45DB161D isadded in this system. The chip is used to store the parameters of $K_{p}, K_{i}, K_{d}$, and the zero value of the position to achieve the reading of the system boot initialization and the writing in the debugging process In addition, it also can be used to store exceptional information during the work process to facilitate futureanalysis and maintenance.

\section{Master Software Design}

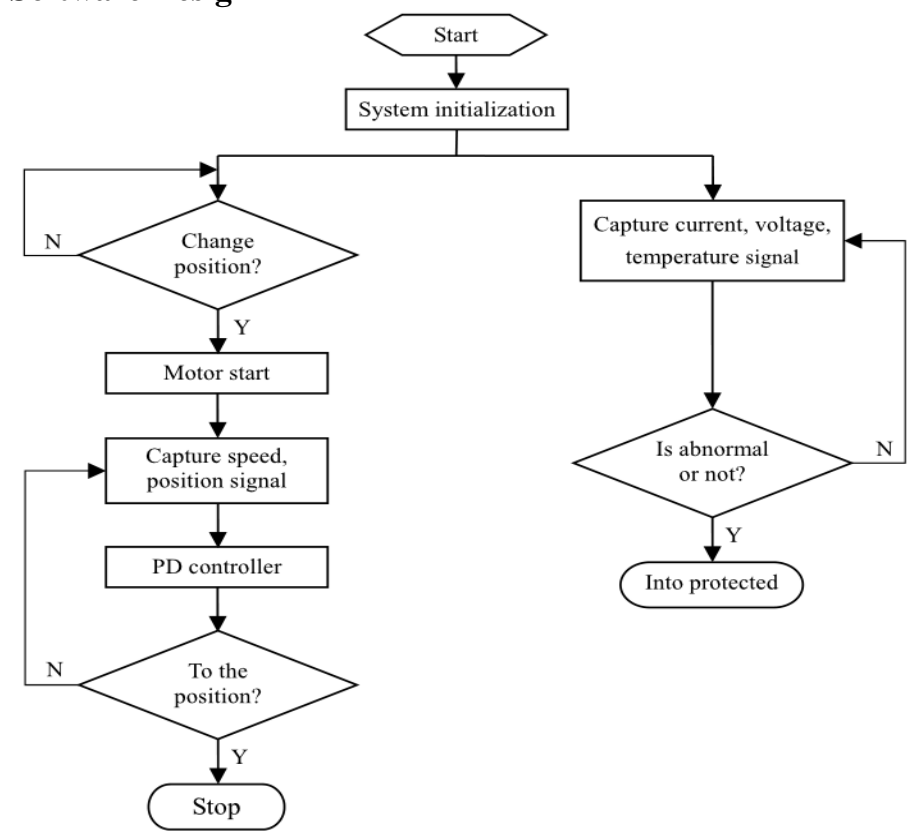

Fig.5. The flow chart of the control program 
The system monitors and protectsthe voltage, current, and temperature while controlling the performing position to ensure the system's reliability.

For the brushless DC motor, single-loop position control mode can be used to allow static error and direct design ofthe position PD regulator [5] with the following formula:

$$
\begin{aligned}
& \text { error }=\theta_{m}^{*}-\theta_{m} . \\
& V_{\text {control }}=K_{p} \times \text { error }-A_{\theta} \times K_{d} \times \text { Speed. }
\end{aligned}
$$

Whether or notthe designated location is reached,the Speedis large.

$$
\begin{aligned}
& V_{\text {min }} \leq \mid K_{p} \times \text { error } \mid \leq V_{\text {max }} . \\
& V_{\text {min }} \leq \mid A_{\theta} \times K_{d} \times \text { Speed } \mid \leq V_{\text {max }} . \\
& V_{\text {min }} \leq\left|V_{\text {control }}\right| \leq V_{\text {max }} .
\end{aligned}
$$

When the designated location is reached,the Speedis approximately zero.

$$
V_{\text {control }}=0 \text {. }
$$

Where, $\theta_{m}^{*}$ is the designated location, $\theta_{m}$ is the current location, Speed is the measured motor speed, $K_{p}$ is a proportionality coefficient, and $K_{d}$ is a differential coefficient. When the motor is close tothe specified location, the motor speed is reduced, thereby resulting in hiddendifferential linksfunctions. Moreover, the motor speed cannot be quickly reduced to zero, resulting in the overshooting of the motor. To solve this problem, the function $A_{\theta}$ isaddedtothe differential links. The function $A_{\theta}$ is an inverse function relating to error. This is used to increase the function of thedifferential decelerationlinks when the motor is nearthe specified location toprevent overshoot deceleration. Because the brushless motor operating voltage is within a certain range, $V_{\min } \sim V_{\max }$ is the voltage region where the measured speed of the brushless motor can smoothly change.To makethe motor rotate continuously when the specified position is not reached or reachedand when the Speed is relatively large, the output amplitudeshould be limited.

The measurement of the current angular position of the motor uses information fusion technology. The relative rotation angle of the motor can be obtained by integrating the Hall sensor output, with a low measurement noise and fast response characteristics, but some steps may be lost. The motor output 
shaft-driven potentiometer directly measures the absolute angular position in the presence of measurement noise and nonlinear during the mechanical transmission and analog acquisition processes. By fusingthe Hall sensor output of the integrator and the potentiometer-measured value information, the Hall sensor output of the integrator can be obtained mainly under these dynamic conditions. Thus, the long cycle position will be corrected with the potentiometer-measured values. Thus, a motor current angular position with low noise, fast response, and goodlinearity can be obtained.

\section{Results}

The system prototype isstep and sinusoidalresponse tested. By continuously adjusting the $K_{p}$ and $K_{d}$ parameter values, the best response curvecan be obtained.

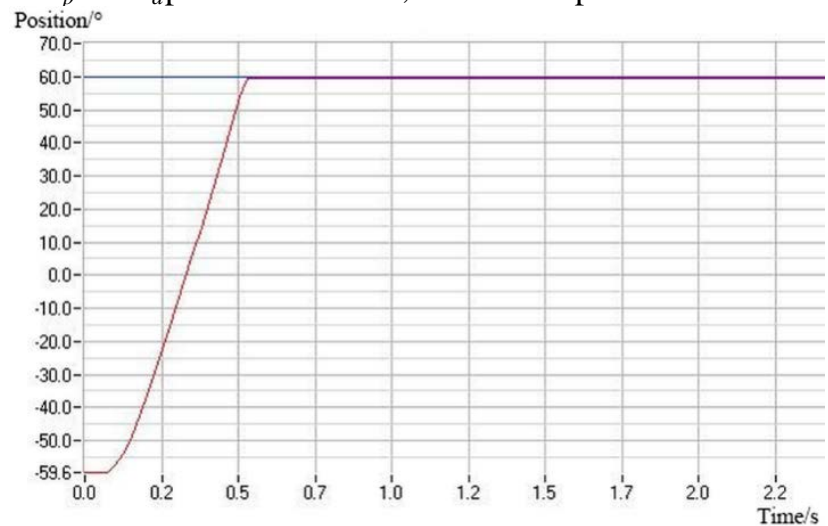

Fig.6. Step signal test curve

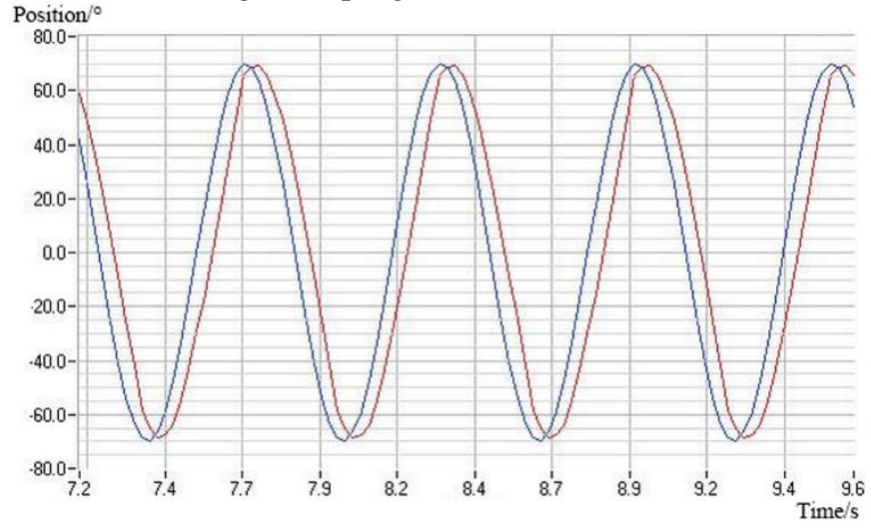

Fig.7. Sine signal test curve

As shown bythetwo figures, this system has good positioning and followingability. The system can achieve non-overshoot or small overshoot and can smoothly follow the command signalof the sinusoidal test.

\section{Conclusions}


The position control algorithm based on the angular position information fusion technology significantly improvesthe step and sinusoidal response characteristics of the prototype as shown in the satisfactory test results.The complete hardware and software protection measuresalso laya solid foundation for the reliable and stable work of the prototype. The prototype of this system has been successfully applied in the engine thrust vector control of the unmanned surface craft project. Good experimental resultshave been obtained from the abovementioned project.

\section{References}

[1] Jun Peng, Fudan Du, Boliang Guan, in: The DSP Control in PMBLDC Motor,Mechatronics Vol. 2 (2003), p. 49-52

[2] Yonghong Wang, Wei Xu, LipingHao: The Principle and Practice of STM32 Series ARM Cortex-M3 Microcontroller(Beihang University Press, China 2008)

[3] Wenfu Qin, Kunfeng Zhang, in: Design of Driver Circuit for BLDCM Based on IR2136, Electronic Design Engineering Vol. 20 (2012), p. 118-120

[4] Jianan Zeng, Yuenan Zeng, MianhaoJi, in: MOSFET and IGBT Drivers IR2136 and Its Application, Drive \& Control Vol. 1 (2005), p. 13-15

[5] Jinfei Li, Haixiao Yuan, Zhihui Chen, in: Study on Position Control of Brushless DC Servo System,Electric Drive Vol. 39 (2009), p. 10-13 